\title{
Discovery of synthetic lethal interactions from large-scale pan-cancer perturbation screens
}

Sumana Srivatsa ${ }^{1,2 \dagger}$, Hesam Montazeri ${ }^{3 \dagger}$, Gaia Bianco ${ }^{4}$, Mairene Coto-Llerena ${ }^{4}$, Charlotte KY $\mathrm{Ng}^{5}$, Salvatore Piscuoglio ${ }^{4,6,7}$ and Niko Beerenwinkel $\mathrm{l}^{1,2^{*}}$

† Contributed equally

* Correspondence to Niko Beerenwinkel niko.beerenwinkel@bsse.ethz.ch

1 Department of Biosystems Science and Engineering, ETH Zurich, 4058 Basel, Switzerland 2 SIB Swiss Institute of Bioinformatics, 4058 Basel, Switzerland

3 Department of Bioinformatics, Institute of Biochemistry and Biophysics, University of Tehran, Tehran, Iran

4 Institute of Pathology, University Hospital Basel, 4031 Basel, Switzerland

5 Department for BioMedical Research, University of Bern, 3008 Bern, Switzerland

6 Visceral Surgery Research Laboratory, Clarunis, Department of Biomedicine, University of Basel, Basel, Switzerland

7 Clarunis Universitäres Bauchzentrum Basel, Basel, Switzerland.

Keywords: SLIdR, Synthetic lethality (SL), perturbation screen, driver genes, SL partner, causal inference, AXIN1-URI1 
Despite the progress in precision oncology, development of cancer therapies is limited by the dearth of suitable drug targets ${ }^{1}$. Novel candidate drug targets can be identified based on the concept of synthetic lethality (SL), which refers to pairs of genes for which an aberration in either gene alone is non-lethal, but co-occurrence of the aberrations is lethal to the cell. We developed SLIdR (Synthetic Lethal Identification in $\underline{R}$ ), a statistical framework for identifying SL pairs from large-scale perturbation screens. SLIdR successfully predicts SL pairs even with small sample sizes while minimizing the number of false positive targets. We applied SLIdR to Project DRIVE data ${ }^{2}$ and found both established and novel pan-cancer and cancer type-specific SL pairs. We identified and experimentally validated a novel SL interaction between AXIN1 and URI1 in hepatocellular carcinoma, thus corroborating the potential of SLIdR to identify new SL-based drug targets.

Key to exploiting SL in cancer therapy is the identification of a targetable dependent gene (SL partner) for a given genetically altered gene, such that a loss-of-function aberration in either gene alone does not affect cell viability, but aberrations in both genes are fatal to the cell (Fig. 1a). A classical example of $S L$ in cancer therapy is the use of PARP inhibitors in BRCA-mutated cancers. The BRCA1/2 genes involved in DNA double-strand break repair are often mutated in breast and ovarian cancers $^{3-5}$, and hence such cancer cells rely on alternate DNA repair processes. PARP1 plays a central role in these alternate DNA repair mechanisms $s^{6,7}$, and therefore inhibiting PARP results in catastrophic double-strand breaks during replication, ultimately leading to cancer cell death ${ }^{8,9}$.

In recent years, large-scale perturbation screens based on siRNA, shRNA, CRISPR, or small molecules in cell lines and organoids have been used to identify SL interactions. McDonald et 
al. ${ }^{2}$ conducted a large-scale deep RNAi screen targeting 7,837 genes in 398 Cancer Cell Line Encyclopedia $^{10}$ (CCLE) models and provided a rich and robust dataset for the identification of SL pairs. However, the authors primarily analyzed gene interactions in a pan-cancer manner. Although pan-cancer analyses are statistically powerful due to their large sample sizes, the underlying data are diverse. We hypothesized that such rich large-scale perturbation screens can be exploited further to obtain SL pairs. We developed a novel method called SLIdR ( Synthetic Lethal Identification in $\underline{R}$ ) for predicting SL partners from such screens in both pan-cancer and cancer type-specific settings.

To define the set of genetically altered genes, we focused on significantly mutated genes reported by MutSig $2 \mathrm{CV}$ v3.111,12 for each cancer type, and considered these genes to be altered in cell lines if they were subject to non-synonymous mutations or deep deletions (Online methods). We collectively refer to these altered genes as driver genes and these alterations as mutations. SLIdR aims to find SL partners for such drivers from perturbation data. We applied SLIdR to the Project DRIVE dataset ${ }^{2}$, focusing on cell lines from $\operatorname{CCLE}^{10}$ with available copy number data across various cancer types (Fig. 1b).

SLIdR adopts a rank-based statistical framework to robustly identify SL interactions between a driver gene and a perturbed gene (Fig. 1c). In contrast to previous methods which perform statistical tests on the raw viability readouts ${ }^{2,13}$, SLIdR uses the normalized ranks of the viabilities across all perturbed genes for each cell line in order to increase statistical power for small sample sizes. For each driver gene, SLIdR first stratifies the cell lines into mutated and wild-type based on the mutation status of the driver gene. Subsequently, it tests, for each perturbed gene, whether the perturbation results in lower ranked viabilities in the mutated cell 
lines but not in the wild-type cell lines. SLIdR uses two Irwin-Hall tests to mine for such driver-perturbed SL gene pairs (Online methods). Cell lines with several co-occurring driver mutations can yield multiple SL pairs with the same perturbed gene. To identify the most likely SL pairs, we perform causal inference using matching-based potential outcome models. For a given candidate pair, we match the wild-type to mutated cell lines based on the other co-occurring mutations, thus achieving a covariate balance. Finally, SLIdR compares the viabilities of the matched groups and the significant SL pairs are reported (Fig. 1d; Online methods).

We first applied SLIdR to the DRIVE data in a pan-cancer setting. We identified 151 SL pairs (Supplementary Table S1) involving 84 driver genes (Fig. 2a). Out of the 151 SL pairs, five pairs involving bona fide driver genes TP53, KRAS, BRAF, CTNNB1, and PIK3CA exhibited self-dependency, i.e., paired with themselves as the SL partner gene. This proved to be an efficient quality check for our method as these are well-established drivers and their subsequent knockdown resulted in cellular mortality. We also found that some cell lines with several co-occurring mutations resulted in multiple driver genes pairing with the same SL partner (Fig. 2b; Supplementary Fig. S1a). For example, co-deletion of genes near p16 including MTAP and several interferons is common in several cancers and subsequently all these drivers paired with MAT2A as the SL partner. Using causal inference, we predicted the relevant driver genes for each SL partner (Fig. 2c; Supplementary Fig. S1b), resulting in 90 SL pairs across 42 driver genes (Fig. 2d).

Top predictions of SLIdR included PRMT5, MAT2A, and RIOK1 as SL partners of MTAP which are all well-established vulnerable targets for MTAP-altered cells ${ }^{2,14}$. SLIdR also predicted E2F3 
and SKP2 as SL partners for $R B 1^{2}$. Furthermore, RPL22 showed lethality with its paralog RPL22L1 confirming the findings of McDonald et al. ${ }^{2}$. PIK3CA-BIRC5 was another reassuring pair as depletion of survivin (BIRC5) has been shown to have a pro-apoptotic effect in breast cancer cells with PIK3CA mutations ${ }^{15,16}$. In addition to established pairs, SLIdR also predicted several new SL pairs such as KRAS-TRPM7 and TP53-USP28, which require further validation (Supplementary Table S2).

While pan-cancer analyses are favoured for their large sample sizes and the ability to identify shared targets across different cancer types, it is often difficult to identify such targets due to inherent differences between primary sites. The differential sensitivities of the predicted pan-cancer hits based on primary sites suggests that the majority of the signal is cancer type-specific (Fig. 2d). For example, SLIdR identified NFE2L2 as the SL partner of the mutated KEAP1, both of which play an important role in cancer through Nrf2 pathway activation ${ }^{17}$. However, we noted that this SL interaction was largely due to signal from lung cancer samples (Fig. 2d), in accordance with Leiserson et al. ${ }^{18}$ who also reported the pair to be mutually exclusive in their pan-cancer TCGA analysis largely due to lung cancer samples. Thus, although a considerable number of pan-cancer hits are consistent with previous findings, this example among several others shows the need to identify cancer type-specific SL partners.

Consequently, we applied SLIdR to the DRIVE data in a cancer type-specific setting. For 17 cancer types, we identified a total of 839 SL pairs (Supplementary Table S1) over 233 unique driver genes. Out of the 233 drivers, 66 genes were mutated in more than one cancer type (Fig. 3a). However, the mutation profiles are diverse across cancer types, with TP53 mutations being highly prevalent and observed in $\sim 81 \%$ of the cancer types, while well-known drivers such as 
$B R A F, A P C$, and PTEN were distinctly associated with skin, large intestine, and endometrial cancers, respectively.

Upon extensive literature survey of the SL pairs we identified 55 established and potential pairs with literature support (Fig. 3b; Supplementary Table S2). For example, SLIdR predicted GATA3-ESR1 in breast cancer. GATA3 is mutated in $>10 \%$ of breast cancers and directly impacts ESR1 enhancer accessibility, thereby altering binding potential and transcriptional targets in tumor cells ${ }^{19}$. Furthermore, GATA3 mutations are almost never observed in ER-negative breast cancers, strongly suggesting SL. Despite the small sample size (7 cell lines), SLIdR also successfully elicited the RB1-MCL1 pair in osteosarcomas. Loss-of-function RB1 mutations are common in osteosarcomas ${ }^{20,21}$ and inhibition of MCL1 has been shown to block tumor growth in osteosarcoma ${ }^{22}$. Additionally, SLIdR predicted several significant SL partners specific to TP53, including TP53BP1, USP28, DDX3, and PNPLA6 in the pan-cancer setting, and HMGA1, RAB14, and RAC1 in osteosarcoma, renal, and breast cancers, respectively (Fig. 3b; Supplementary Table S2). These examples highlight the ability of SLIdR to identify well-established and novel targets in both pan-cancer and cancer type-specific settings.

In hepatocellular carcinoma (HCC) we identified nine SL pairs (Fig. 3c). To demonstrate the predictive power of SLIdR, we sought to validate AXIN1-URI1 (Fig. 4a), a novel pair and our top prediction in HCC. First, we validated the SL interaction between AXIN1 and URI1 in vitro using SNU449, a HCC cell line carrying an AXIN1 somatic mutation. Upon confirming that silencing of URI1 using siRNA reduced URI1 mRNA expression by $>50 \%$ up to 96 hours post-transfection (Fig. 4b), we assessed cell viability by measuring cell proliferation rate. We observed that the 
knockdown of URI1 in SNU449 cells significantly decreased proliferation compared to control cells (Fig. 4c).

Next, we validated the SL interaction between AXIN1 and URI1 in Huh-7, an AXIN1-wildtype HCC-derived cell line. In this cell line, we silenced AXIN1 and UR/1 alone or in combination. After confirming that silencing of the genes using siRNAs reduced their mRNA levels by $50-90 \%$ up to 96 hours post-transfection (Fig. 4e), we checked cells for growth inhibition using the same experimental approaches previously used in SNU449 (Figs. 4d,e). Huh-7 cells transfected with siRNAs targeting both URI1 and AXIN1 proliferated significantly less compared to cells transfected with CTRL siRNA, AXIN1 siRNA or URI1 siRNA alone (Fig. 4d). By staining cells with Annexin $\mathrm{V}$ and propidium iodide and analysing them by FACS, we showed that cells transfected with both AXIN1 and URI1 siRNA showed a higher proportion of apoptotic cells (15-20\% more) and a lower proportion of living cells ( $20 \%$ less) compared to CTRL cells and cells transfected with either URI1 or AXIN1 siRNA alone (Fig. 4f), demonstrating that dual silencing was indeed fatal to the cells rather than merely arresting their proliferation.

To ensure that the cell death was truly due to synthetic lethality between AXIN1 and URI1 and not a result of an off-target effect or the cumulative cytotoxicity of the double siRNA transfection, we used the non-SL AXIN1-TP53 pair as a negative control (Supplementary Fig. S2a). Dual silencing of AXIN1 and TP53 did not result in decreased cell proliferation compared to silencing of TP53 or AXIN1 alone (Supplementary Fig. S2c), confirming AXIN1-URI1 as a novel SL pair in HCC. 
bioRxiv preprint doi: https://doi.org/10.1101/810374; this version posted October 18,2019 . The copyright holder for this preprint (which was not certified by peer review) is the author/funder, who has granted bioRxiv a license to display the preprint in perpetuity. It is made available under aCC-BY-NC-ND 4.0 International license.

Taken together, SLIdR provides a robust statistical framework to facilitate rapid discovery of SL interactions from large-scale perturbation screens in both pan-cancer and cancer type-specific settings. Particularly, in precision oncology, SLIdR can help in developing novel mutation-specific and effective personalised therapies. 


\section{ONLINE METHODS}

\section{Screening data}

We used viability profiles published in Project DRIVE ${ }^{2}$, as well as corresponding mutation data and copy number data from the Cancer Cell Line Encyclopedia (CCLE) collection ${ }^{10}$ for 373 cell lines across 23 cancer types.

\section{Viability data from perturbation screens}

Viability data specifies the viability of cell lines for each gene knockdown experiment. In Project DRIVE, 7837 genes were targeted by using an average of 20 pooled shRNAs per gene. The shRNA activities were defined as the quantile normalised log fold change in shRNA read counts 14 days after the start of the knockdown experiment to the shRNA abundance in the input library. The gene-level viability score of each cell line was computed by aggregation of shRNA activities using two computational methods, namely $\mathrm{RSA}^{23}$ and ATARiS ${ }^{24}$. The RSA method uses all shRNA reagents targeting a gene and can be used to identify essential, inert and active genes, while ATARiS only uses a subset of shRNAs with consistent activity across the cell lines and aims to provide a robust gene-level score by discarding shRNA reagents with off-target effects. ATARiS provides a relative score for the gene-level activity by median-centering the data for each reagent, and as a result, cannot distinguish between inert and essential genes.

To process the viability data, we removed essential genes using the RSA method as was performed in Project DRIVE ${ }^{2}$. Genes with an RSA value $\leq-3$ in more than $50 \%$ of cancer cell lines were reported as essential genes. In total, 460 and 185 genes were reported essential in cancer type-specific and pan-cancer settings, respectively. The resulting viability matrices 
consisted of the ATARiS scores for the remaining perturbed genes (rows) for each cell line (columns).

\section{Mutation and copy number data}

For the pan-cancer setting, we focused on genes with mutations or copy-number aberrations in more than 30 cell lines. We downloaded mutation data and copy number data from the CCLE website, and binarized them as follows. A gene in a given cell line was assigned a value of 1 if it was subject to non-synonymous mutations, and 0 otherwise. For copy number data, we focused only on homozygous deletions and binarized a gene in a given cell line by assigning a value of 1 if the gene was homozygously deleted and a value of 0 otherwise. Finally, combining both these data, a driver gene in a given cell line was assigned a value of 1 if it was subject to non-synonymous mutations, deep deletions, or both; 0 otherwise.

In the cancer type-specific setting, to define the set of driver genes, we first used the MutSig 2CV v3.1 $1^{11,12}$ MAF file from TCGA for each cancer type and focused only on significantly mutated genes $(q<=0.05)$. Next, we concentrated on genes with non-synonymous mutations in two or more cell lines, and excluded copy number data as it was very noisy in this setting. Thus, a gene in a given cell line was assigned a value of 1 if it was subject to non-synonymous mutations, and 0 otherwise. The resulting binarised mutation matrices described the mutation profiles for each cell line (column) across all driver genes (rows).

\section{SLIdR algorithm}

SLIdR is a rank-based statistical framework to identify the presence of synthetic lethal dependency between a driver gene $d$ and a perturbed gene $g$. For each driver gene $d$, we 
divided the cell lines into two groups according to the mutation status of $d$, namely wild-type cell lines (WT) and mutated cell lines (Mut). Further, we ranked the perturbed genes by their ATARiS scores, for each mutated and WT cell line and normalized it between 0 and 1 . Due to a large number of perturbed genes, the normalized ranks have many distinct levels and are highly fine-grained. Hence, we assumed the normalized ranks to be continuous.

Let $(d, g)$ be a fixed pair of driver and perturbed gene and $C_{d}$ be the set of cell lines mutated in $d$ of cardinality $n$. If $(d, g)$ is an SL pair, based on the aforementioned definition (Fig. 1a), a mutation in driver gene $d$ in combination with knockdown of gene $g$, results in low viabilities in mutated cell lines $C_{d}$. We used a one-sided statistical test based on the Irwin-Hall distribution to test whether the viabilities of mutated cell lines $C_{d}$ from knockdown of gene $g$ are lower than expected by chance. We defined the null hypothesis $H_{0}$ as the knockdown of gene $g$ having no impact on the viability of the cell lines in $C_{d}$. For each cell line $c \in C_{d}$, we computed the normalized rank of the viability of $c$ from knockdown of gene $g$ across all other gene knockdowns in cell line $c$, and denoted this rank as $r_{c \mid g}$. Under the null hypothesis, the normalized ranks take uniform random values in the interval $[0,1], r_{c \mid g} \sim U(0,1)$. The test statistic $T$ for the pair $(d, g)$ is then defined as the sum of normalized viability ranks of mutated cell lines $C_{d}$ perturbed in gene $g, T=\sum_{c \in C_{d}} r_{c \mid g}$. Under $H_{0}$, the test statistic $T$ is the sum of $n$ independent uniform random variables on the unit interval and hence it follows the Irwin-Hall distribution of order $n$. The resulting $\mathrm{p}$-value was computed as the lower tail probability $P\left(T<t_{o b s}\right)$, where $t_{o b s}$ is the observed test statistic. For large $n$, computation of the Irwin-Hall probability distribution is either computationally expensive or numerically unstable. Therefore, we used the approximation $T \sim N(n / 2, n / 12)$ for $n>20$. 
Conversely, based on the definition of synthetic lethality (Fig. 1a), wild-type cell lines with respect to driver gene $d$ are expected to behave similar to healthy cells when perturbed in gene $g$. Therefore, it is important to filter out genes which upon knockdown adversely alter the viabilities of WT cell lines. We used a two-sided Irwin-Hall test to filter out any pair $(d, g)$ that reached statistical significance ( $\alpha=0.1$ ) in the WT cell lines. However, we did not use this filter for pan-cancer setting due to the diverse nature of the cell types and cancer types.

\section{Multiple testing}

We reduced the number of false positives arising from multiple testing by choosing a significance level of $1 /(M \times N)$, where $M$ is the number of knockdowns and $N$ is the number of driver genes. Therefore, we expect on average one false positive hit among all reported SL hits for each cancer. Our method is computationally inexpensive as it avoids performing all $M \times N$ tests. For each driver, we compute the test statistic for all perturbed genes and sort them in ascending order. The pre-ordering of the test statistics enables us to test for genes until the corresponding $p$-value is less than the chosen significance level. Further, we note that this approach was in good agreement with controlling the false discovery rate at $10 \%$ (Supplementary Fig. S1c).

\section{Causal inference}

Cell lines are often subject to mutations or aberrations in multiple driver genes, and as a result, different driver genes pair with the same SL partner gene (Fig. 2b; Supplementary Fig. S1a). This is typically not an issue in the cancer type-specific setting but is prevalent in the pan-cancer setting. In order to identify the most likely SL pairs from the many confounding driver genes, we 
used matching-based potential outcome models. The main goal of the matching method is to emulate a randomized experiment by matching samples of treated and control groups according to covariates, thereby obtaining similar covariate distributions across the two groups. For a given driver gene $d$, the cell lines mutated in $d$ constituted the treated group and the cell lines wild-type in $d$ formed the control group. The remaining driver genes formed the confounding covariates and the viability of the SL partner gene $g$ was used as the response or outcome variable. We used the Matching $\mathrm{R}$ package $\mathrm{e}^{25}$ and performed propensity-score matching with a caliper of 0.1 . Since matching is dependent on the order of the samples, we reshuffled and repeated matching 50 times. After each run, we recorded the standardised mean difference (smd) between the two groups for all covariates and chose the run with the lowest sum of smd across all covariates. Finally, for the chosen run, we performed a paired t-test between the responses of treated and control groups. We repeated this entire process for all the driver genes pairing with the same SL partner gene $g$ and reported the significant $(\alpha=0.05)$ SL pairs (Fig.

\section{2c; Supplementary Fig. S1b).}

\section{Code and data availability.}

The code base of SLIDR is available at https://github.com/cbg-ethz/slidr/. The raw shRNA data has already been published as a part of project DRIVE (https://data.mendeley.com/datasets/y3ds55n88r/4) and all the mutation and copy number data from CCLE is available at https://portals.broadinstitute.org/ccle. The MutSig 2CV v3.1 $1^{11,12} \mathrm{MAF}$ file for each cancer type is available at http://firebrowse.org/.

\section{Cell lines maintenance}


Liver cancer-derived cell lines SNU449 and Huh-7 were obtained from the Laboratory of Experimental Carcinogenesis (Bethesda, MD, USA), authenticated by short tandem repeat profiling as previously described ${ }^{26}$ and tested for mycoplasma infection using a PCR-based test (ATCC). All cell lines were maintained under the conditions recommended by the provider. Briefly, all cell lines were cultured in DMEM supplemented with 5\% Fetal Bovine Serum (FBS), non-essential amino-acids (NEAA) and antibiotics (Penicillin/Streptomycin). The cells were incubated at $37^{\circ} \mathrm{C}$ in a humidified atmosphere containing $5 \% \mathrm{CO}_{2}$. Exponentially growing cells were used for all in vitro studies.

\section{Transient gene knockdown by siRNAs}

Transient gene knockdown was conducted using ON-TARGET plus siRNA transfection. ON-TARGET plus SMARTpool siRNAs against human URI1, AXIN1 and TP53, ON-TARGET plus SMARTpool non-targeting control and DharmaFECT reagent were all purchased from GE Dharmacon (Supplementary Table S3). Transfection was performed according to the manufacturer's protocol. Briefly, log-phase liver cancer cells were seeded at approximately $60 \%$ confluence. Since residual serum affects the knockdown efficiency of ON-TARGET plus siRNAs, growth medium was removed as much as possible and replaced by serum-free medium (Opti-MEM; Supplementary Table S3). siRNAs were added to a final concentration of $25 \mathrm{nM}$. siRNAs targeting different genes can be multiplexed. Cells were incubated at $37^{\circ} \mathrm{C}$ in $5 \% \mathrm{CO}_{2}$ for 24-48-72 hours (for mRNA analysis) or for 48-72 hours (for protein analysis). In order to avoid cytotoxicity, transfection medium was replaced with complete medium after 24 hours.

\section{RNA extraction and relative expression by qRT-PCR}


Total RNA and proteins were extracted from cells at $75 \%$ confluence using TRIZOL (Supplementary Table S3) according to manufacturer's guidelines. cDNA was synthesized from $1 \mu \mathrm{g}$ of total RNA using SuperScript ${ }^{\mathrm{TM}}$ VILO $^{\mathrm{TM}}$ cDNA Synthesis Kit (Supplementary Table S3). All reverse transcriptase reactions, including no-template controls, were run on an Applied Biosystem $7900 \mathrm{HT}$ thermocycler. Gene expression was assessed by using FastSart Universal SYBR Green Master Mix (Supplementary Table S3) and all qPCR were performed at $50^{\circ} \mathrm{C}$ for $2 \mathrm{~min}, 95^{\circ} \mathrm{C}$ for $10 \mathrm{~min}$, and then 40 cycles of $95^{\circ} \mathrm{C}$ for $15 \mathrm{~s}$ and $60^{\circ} \mathrm{C}$ for $1 \mathrm{~min}$ on a QuantStudio 3 Real-Time PCR System (Applied Biosystems). The specificity of the reaction was verified by melting curve analysis. Measurements were normalized using GAPDH level as the reference. The fold change in gene expression was calculated using the standard $\Delta \Delta \mathrm{Ct}$ method as previously described ${ }^{27}$. All samples were analyzed in triplicates.

\section{Proliferation assay}

Cell proliferation was assayed using the xCELLigence system (RTCA, ACEA Biosciences, San Diego, CA, USA). Background impedance of the xCELLigence system was measured for $12 \mathrm{~s}$ using $50 \mu \mathrm{l}$ of room temperature cell culture media in each well of E-plate 16. Cells were grown and expanded in tissue culture flasks as previously described. After reaching $75 \%$ confluence, the cells were washed with PBS and detached from the flasks using a short treatment with trypsin/EDTA. 5000 cells were dispensed into each well of an E-plate 16. Growth and proliferation of the cells were monitored every $15 \mathrm{~min}$ up to 120 hours via the incorporated sensor electrode arrays of the xCELLigence system, using the RTCA-integrated software according to the manufacturer's parameters. In the case of transient siRNA transfection, cells were detached and plated on xCELLigence 24 hours post-transfection. 


\section{Apoptosis analysis by Flow Cytometry}

Cells were collected 72 hours post siRNA transfection, stained with annexin V (FITC conjugate; Supplementary Table S3) and propidium iodide (PI), and analyzed by flow cytometry using the BD FACSCanto II cytometer (BD Biosciences, USA). Briefly, cells were harvested after incubation period and washed twice by centrifugation $(1,200 \mathrm{~g}, 5 \mathrm{~min})$ in cold phosphate-buffered saline (DPBS; Supplementary Table S3). After washing, cells were resuspended in $0.1 \mathrm{~mL}$ AnnV binding buffer $1 \mathrm{X}$ (ABB 5X, Supplementary Table S3) containing fluorochrome-conjugated $\mathrm{AnnV}$ and $\mathrm{PI}$ (PI to a final concentration of $1 \mathrm{ug} / \mathrm{mL}$ ) and incubated in darkness at room temperature for 15 min. Following immediately, cells were analyzed by flow cytometry, measuring the fluorescence emission at $530 \mathrm{~nm}$ and $>575 \mathrm{~nm}$. Data were analyzed by FlowJo software version 10.5.3 (https://www.flowjo.com/).

For details on the reagents used, please refer to Supplementary Table S3.

\section{ACKNOWLEDGEMENTS \& FUNDING}

This work was partly supported by ERC Synergy Grant 609883 to N.B. Swiss Cancer League [KFS-3995-08-2016] and the Swiss National Foundation [PZ00P3_168165] to S.P.

\section{AUTHOR'S CONTRIBUTION}

S.S. and N.B. conceived the presented idea. S.S. and H.M. developed and implemented the method and built the R package. N.B. supervised the computational study. S.P. and C.K.Y.N. supervised the experimental study. S.S. performed the downstream bioinformatics analyses and contributed to the visualizations. G.B. and M. C-L performed in vitro experiments. N.B., S.P. and C.K.Y.N. critically discussed the results. S.S., H.M., G.B., M. C-L, C.K.Y.N., S.P., and N.B. 
bioRxiv preprint doi: https://doi.org/10.1101/810374; this version posted October 18,2019 . The copyright holder for this preprint (which was not certified by peer review) is the author/funder, who has granted bioRxiv a license to display the preprint in perpetuity. It is made available under aCC-BY-NC-ND 4.0 International license.

interpreted the results and wrote the manuscript. All authors agreed to the final version of the manuscript. 


\section{REFERENCES}

1. Brunen, D. \& Bernards, R. Drug therapy: Exploiting synthetic lethality to improve cancer therapy. Nat. Rev. Clin. Oncol. 14, 331-332 (2017).

2. McDonald, E. R., 3rd et al. Project DRIVE: A Compendium of Cancer Dependencies and Synthetic Lethal Relationships Uncovered by Large-Scale, Deep RNAi Screening. Cell 170, 577-592.e10 (2017).

3. Miki, Y. et al. A strong candidate for the breast and ovarian cancer susceptibility gene BRCA1. Science 266, 66-71 (1994).

4. Wooster, R. et al. Identification of the breast cancer susceptibility gene BRCA2. Nature 378, 789-792 (1995).

5. Powell, S. N. \& Kachnic, L. A. Roles of BRCA1 and BRCA2 in homologous recombination, DNA replication fidelity and the cellular response to ionizing radiation. Oncogene 22, 5784-5791 (2003).

6. Metzger, M. J., Stoddard, B. L. \& Monnat, R. J. PARP-mediated repair, homologous recombination, and back-up non-homologous end joining-like repair of single-strand nicks. DNA Repair 12, 529-534 (2013).

7. Ying, S., Hamdy, F. C. \& Helleday, T. Mre11-Dependent Degradation of Stalled DNA Replication Forks Is Prevented by BRCA2 and PARP1. Cancer Research 72, 2814-2821 (2012).

8. Shaheen, M., Allen, C., Nickoloff, J. A. \& Hromas, R. Synthetic lethality: exploiting the addiction of cancer to DNA repair. Blood 117, 6074-6082 (2011).

9. Bryant, H. E. et al. Specific killing of BRCA2-deficient tumours with inhibitors of poly(ADP-ribose) polymerase. Nature 434, 913-917 (2005).

10. Barretina, J. et al. The Cancer Cell Line Encyclopedia enables predictive modelling of 
anticancer drug sensitivity. Nature 483, 603-607 (2012).

11. Lawrence, M. S. et al. Mutational heterogeneity in cancer and the search for new cancer-associated genes. Nature 499, 214-218 (2013).

12. Lawrence, M. S. et al. Discovery and saturation analysis of cancer genes across 21 tumour types. Nature 505, 495-501 (2014).

13. Jerby-Arnon, L. et al. Predicting cancer-specific vulnerability via data-driven detection of synthetic lethality. Cell 158, 1199-1209 (2014).

14. Marjon, K. et al. MTAP Deletions in Cancer Create Vulnerability to Targeting of the MAT2A/PRMT5/RIOK1 Axis. Cell Rep. 15, 574-587 (2016).

15. Tanizaki, J. et al. Roles of BIM induction and survivin downregulation in lapatinib-induced apoptosis in breast cancer cells with HER2 amplification. Oncogene 30, 4097-4106 (2011).

16. Zhao, P. et al. Regulation of survivin by PI3K/Akt/p70S6K1 pathway. Biochem. Biophys. Res. Commun. 395, 219-224 (2010).

17. Goldstein, L. D. et al. Recurrent Loss of NFE2L2 Exon 2 Is a Mechanism for Nrf2 Pathway Activation in Human Cancers. Cell Rep. 16, 2605-2617 (2016).

18. Leiserson, M. D. M. et al. Pan-cancer network analysis identifies combinations of rare somatic mutations across pathways and protein complexes. Nat. Genet. 47, 106-114 (2015).

19. Theodorou, V., Stark, R., Menon, S. \& Carroll, J. S. GATA3 acts upstream of FOXA1 in mediating ESR1 binding by shaping enhancer accessibility. Genome Res. 23, 12-22 (2013).

20. Kansara, M. \& Thomas, D. M. Molecular Pathogenesis of Osteosarcoma. DNA and Cell Biology 26, 1-18 (2007).

21. Chen, X. et al. Recurrent somatic structural variations contribute to tumorigenesis in 
pediatric osteosarcoma. Cell Rep. 7, 104-112 (2014).

22. Pignochino, Y. et al. Sorafenib blocks tumour growth, angiogenesis and metastatic potential in preclinical models of osteosarcoma through a mechanism potentially involving the inhibition of ERK1/2, MCL-1 and ezrin pathways. Mol. Cancer 8, 118 (2009).

23. König, R. et al. A probability-based approach for the analysis of large-scale RNAi screens. Nat. Methods 4, 847-849 (2007).

24. Shao, D. D. et al. ATARiS: computational quantification of gene suppression phenotypes from multisample RNAi screens. Genome Res. 23, 665-678 (2013).

25. Sekhon, J. S. Multivariate and Propensity Score Matching Software with Automated Balance Optimization: TheMatchingPackage forR. Journal of Statistical Software 42, (2011).

26. Reid, Y., Storts, D., Riss, T. \& Minor, L. Authentication of Human Cell Lines by STR DNA Profiling Analysis. in Assay Guidance Manual (eds. Sittampalam, G. S. et al.) (Eli Lilly \& Company and the National Center for AdvancingTranslational Sciences, 2013).

27. Livak, K. J. \& Schmittgen, T. D. Analysis of Relative Gene Expression Data Using Real-Time Quantitative PCR and the 2- $\Delta \Delta$ CT Method. Methods 25, 402-408 (2001). 
bioRxiv preprint doi: https://doi.org/10.1101/810374; this version posted October 18,2019 . The copyright holder for this preprint (which was not certified by peer review) is the author/funder, who has granted bioRxiv a license to display the preprint in perpetuity. It is made available under aCC-BY-NC-ND 4.0 International license.

a

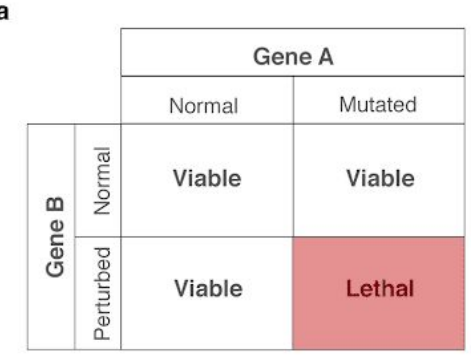

b

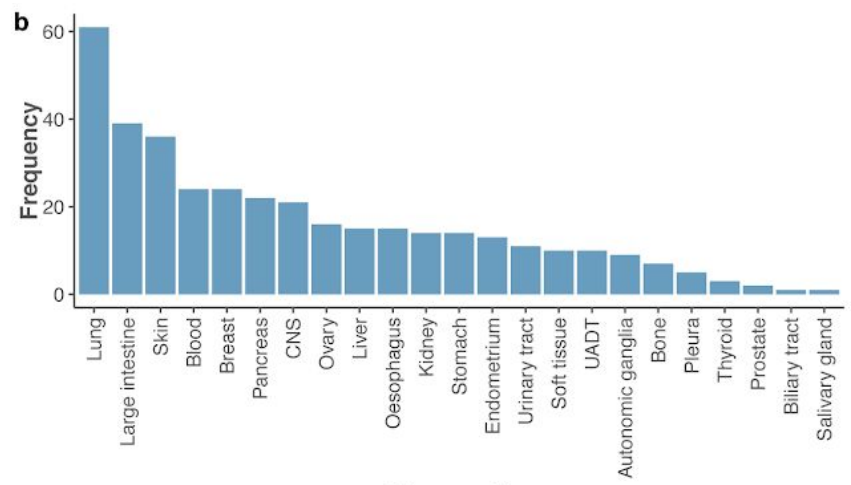

Primary sites

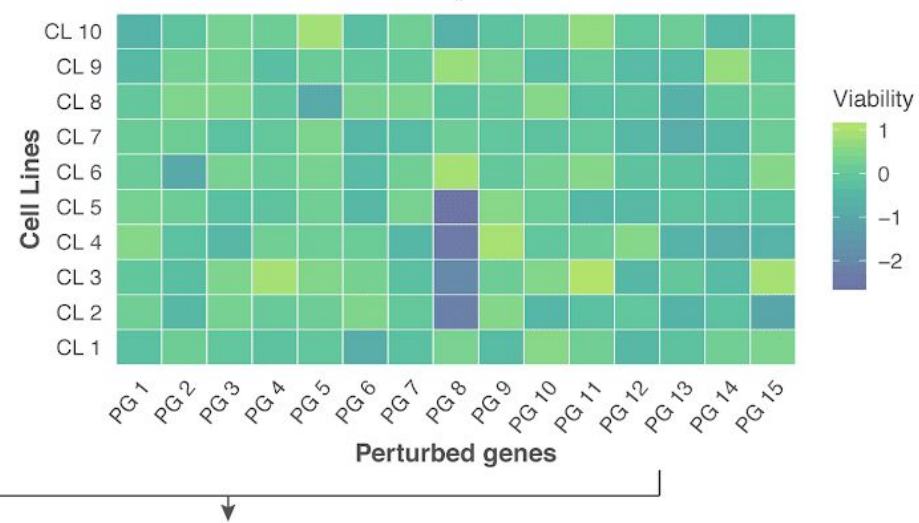

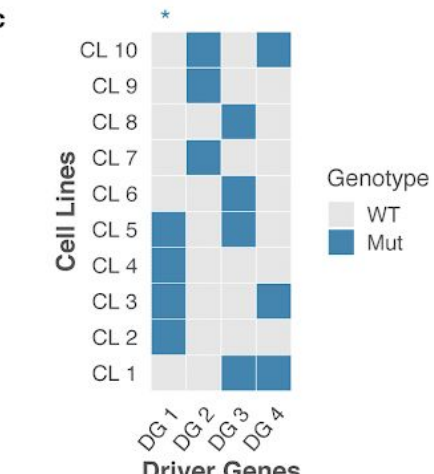

DG 1 - PG 8 SL pair

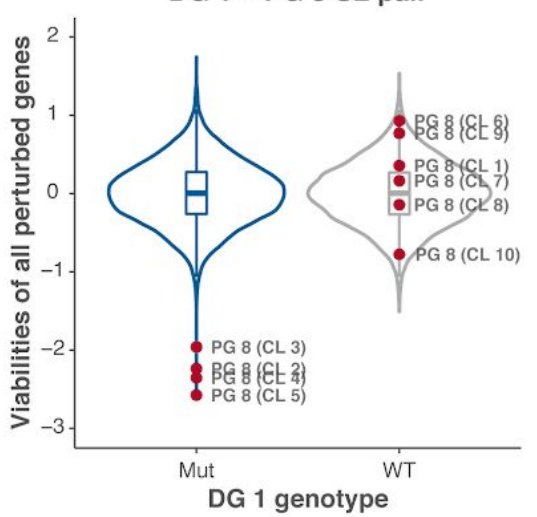

d

\begin{tabular}{l|l|l|l|l|l|l|l|} 
Mutation and \\
Viabilty data
\end{tabular}


Fig. 1. Overview and SLIdR workflow. a, Definition of a synthetic lethal pair: Aberration of gene $A$ or knockdown of gene B alone do not affect the viability of the cell. However, the combination of mutated gene A and knockdown of gene B is lethal to the cell. $\mathbf{b}$, Distribution of the number of cell lines with copy number data from CCLE across 23 different cancer types used in this study. c, Illustration of the SLIdR algorithm with a toy example. The data consists of driver genes DG 1-DG 4 and perturbed genes PG 1-PG 15 across cell lines CL 1-CL 10. Cell lines CL 2-5 are mutated in the driver gene DG 1 (Mut), while the remaining cell lines are DG 1 wild-type (WT). Comparison of viability distributions across all perturbed genes PG 1-PG 15 in the DG 1 mutated (Mut) and WT cell lines shows that perturbation of gene 8 (PG 8) results in reduced viability only in CL $2-5$ and not the WT cell lines. Thus, PG 8 is a SL partner of DG 1. d, The computational pipeline illustrating the different steps performed to obtain the candidate SL pairs from mutation profiles and perturbation screen data. 


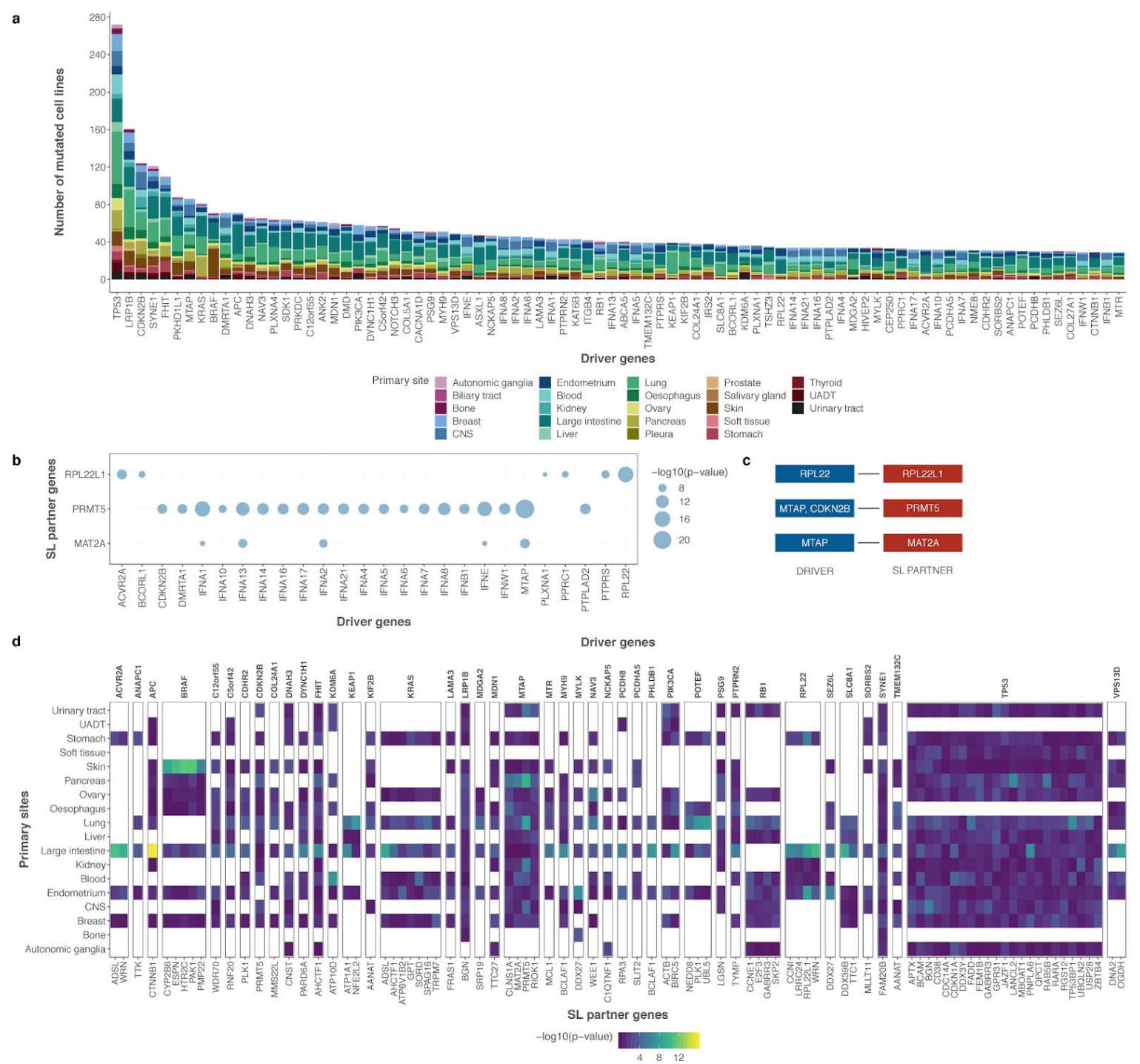

Fig. 2. Pan-cancer SLIdR predictions. a, Stacked barplot indicating the frequencies of 84 mutated driver genes across different cancer types. b, Bubble-plot summarising the significance $(-\log 10(p-v a l u e))$ of different driver genes (x-axis) pairing with the same SL partner gene (y-axis). c, Corresponding list of significant SL pairs after accounting for confounding mutations and performing causal inference using matching-based potential outcome models. d, Differential sensitivities of pan-cancer SL pairs in subsets of cell lines grouped by primary sites (y-axis). Each panel corresponds to a specific driver gene ( $\mathrm{x}$-axis top) and encapsulates the significance 
bioRxiv preprint doi: https://doi.org/10.1101/810374; this version posted October 18, 2019. The copyright holder for this preprint (which was not certified by peer review) is the author/funder, who has granted bioRxiv a license to display the preprint in perpetuity. It is made available under aCC-BY-NC-ND 4.0 International license.

profiles of all its SL-partners (x-axis bottom). Each column in a given panel depicts the significance profile of the SL pair in subsets of cell lines grouped by primary sites. The p-values are computed using $\mathrm{IH}$-test.
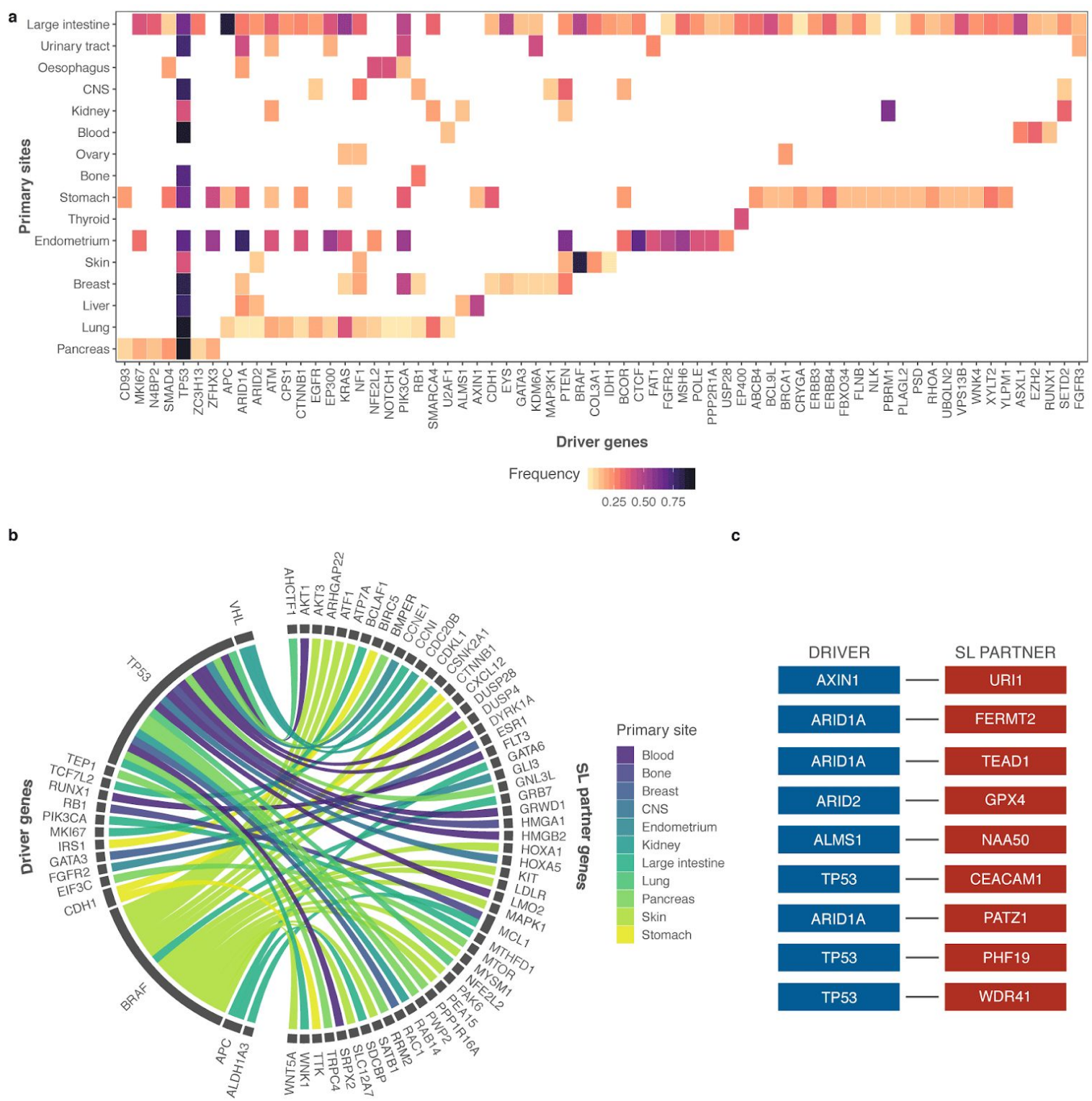

\begin{tabular}{|c|c|}
\hline DRIVER & SL PARTNER \\
\hline AXIN1 & URI1 \\
\hline ARID1A & FERMT2 \\
\hline ARID1A & TEAD1 \\
\hline ARID2 & GPX4 \\
\hline ALMS1 & TAA50 \\
\hline TP53 & CEACAM1 \\
\hline ARID1A & CEAT1 \\
\hline TP53 & PAT2 \\
\hline TP53 & PHF19 \\
\hline
\end{tabular}


Fig. 3. Cancer type-specific SLIdR predictions. a, Heatmap of frequencies of 66 driver genes across 16 cancer types. b, Circos plot summarizing the SL partners (right) of different driver genes (left) with literature evidence, across 11 cancer types. c, Top-ranked SL pairs in hepatocellular carcinoma reported by SLIdR.

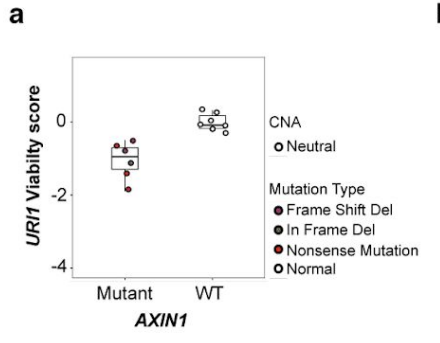

d

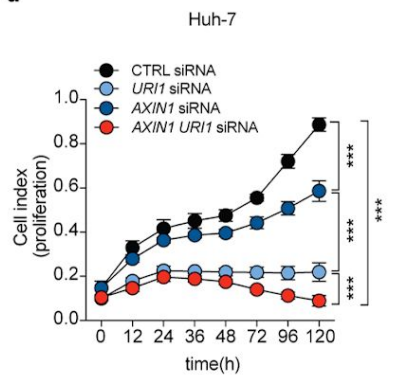

b

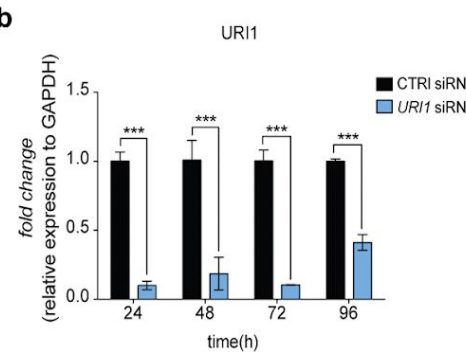

C

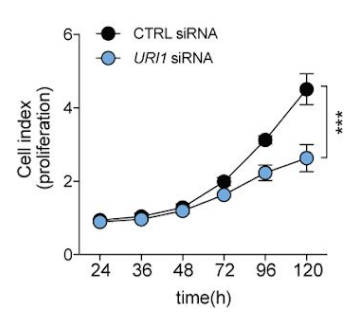

e
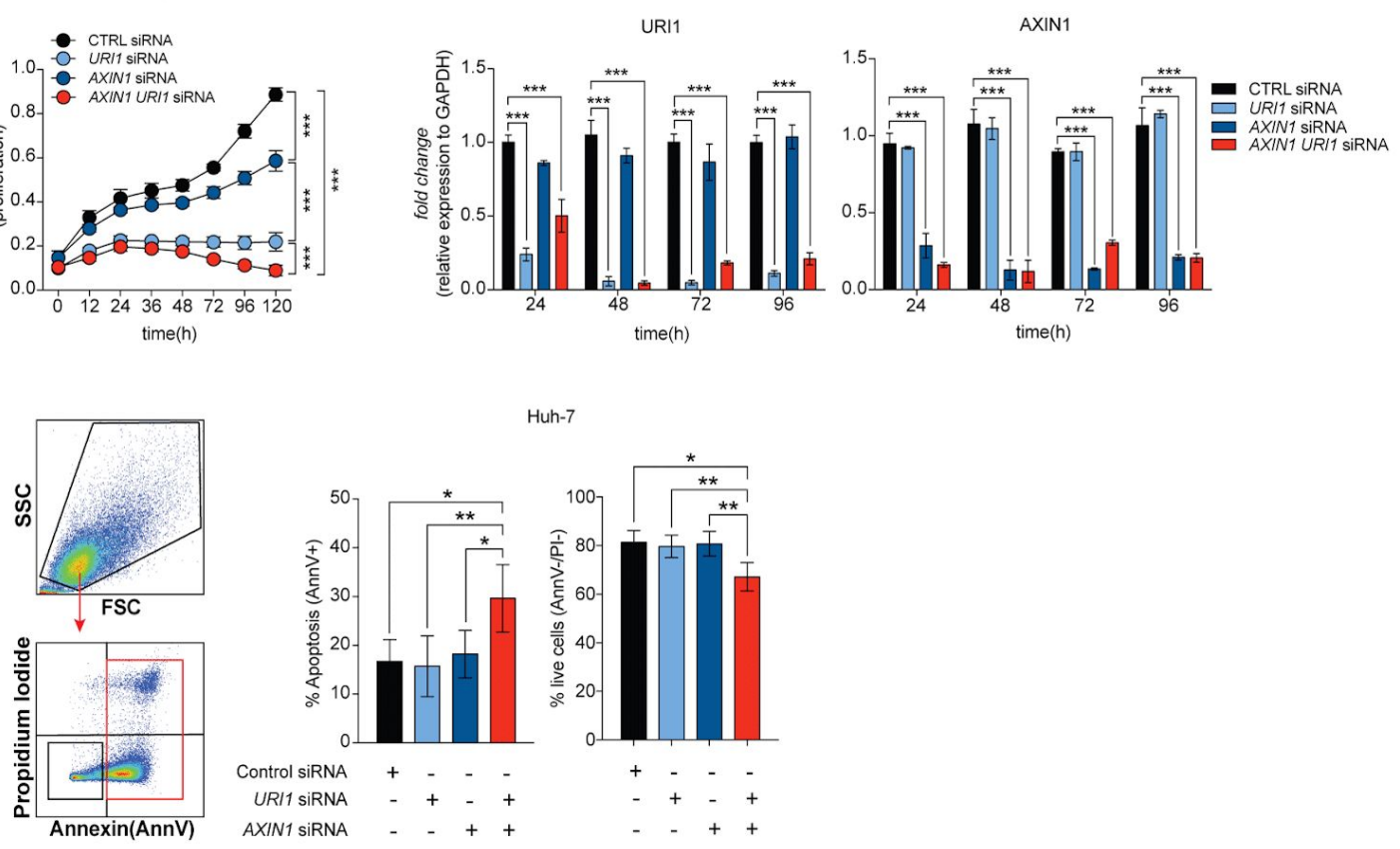

Fig. 4. Functional validation of SL interaction between AXIN1 and URI1. a, Top SL pair in HCC. Viability scores of AXIN1 mutant vs wild-type (WT) HCC cell lines with URI1 knockdown. 
b, RNA expression level (fold-change) of UR/1 relative to GAPDH in SNU449 cells transfected with control siRNA (black) or URI1 siRNA (light blue). RNA levels were assessed by quantitative real-time PCR (qPCR). Error bars represent SD from two independent experiments. c, Cell proliferation assay in SNU449 cell line (AXIN1 mutated) transfected with control siRNA (black) or URI1 siRNA (light blue). Error bars represent standard deviation (SD) from two independent experiments. d, Cell proliferation assay in Huh-7 cell line (AXIN1 WT) transfected with control siRNA (black), URI1 siRNA (light blue), AXIN1 siRNA (dark blue) or both (red). Error bars represent SD from three independent experiments. e, RNA expression levels (fold-change) of URI1 (left) and AXIN1 (right) relative to GAPDH in Huh-7 cell line transfected with control siRNA (black), URI1 siRNA (light blue), AXIN1 siRNA (dark blue) or both (red). Error bars represent SD from three independent experiments. f, Apoptosis assay using Annexin $V$ and propidium iodide (PI) staining in Huh-7 cell line (AXIN1 wild-type) transfected with control siRNA (black), URI1 siRNA (light blue), AXIN1 siRNA (dark blue) or both (red). Quantification of the mean (+/- SD) percentage of apoptotic cells (AnnexinV+) and live cells (PI-/AnnexinV-) across the different groups $(n=4)$ (right). Error bars represent SD from two independent experiments. For all experiments performed, statistical significance was assessed by multiple t-tests ( ${ }^{*} P<0.05$, ${ }^{* *} P$ $\left.<0.01,{ }^{* * *} P<0.001\right)$. 